\title{
Cingulate Sulcus Development in Preterm Infants ${ }^{1}$
}

\author{
TERRI A. SLAGLE, MICHAEL OLIPHANT, AND STEVEN J. GROSS
}

Departments of Pediatrics and Radiology, State University of New York-Health Science Center at Syracuse, Syracuse, New York 13210

\begin{abstract}
Cranial ultrasounds performed during the first $3 \mathrm{~d}$ of life on 211 infants of 24 to 40 wk gestational age were examined to determine the in utero development of the cingulate sulcus. The sulcus was identified between 24 and 28 wk of gestational age as fragmented echoes in the region between the thalamus and the anterior fontanelle. Over the next several weeks, these fragments coalesced into a single linear echo. Branches then appeared off of the primary cingulate sulcus, increasing in number until a complex pattern of branching was noted near term gestation. This maturational sequence was similar to postnatal cortical development determined from serial ultrasounds performed on 144 infants who were $\leq 32 \mathrm{wk}$ of gestational age at birth. The timing of postconceptional cingulate sulcus development was independent of gestational age at birth. However, severe brain insult, defined as intraventricular hemorrhage complicated by ventriculomegaly or intraparenchymal extension or periventricular leukomalacia, was associated with significant delays in all stages of cingulate sulcus development. Cranial ultrasound examinations in preterm infants allow a noninvasive means of staging qualitative brain development during the early postnatal period. (Pediatr Res 26: 598-602, 1989)
\end{abstract}

The human brain undergoes rapid growth and development during the perinatal period and consequently is most vulnerable to the effects of growth restriction at this time (1). Poor brain growth as reflected by less head circumference growth during this critical period of development may have profound effects on subsequent neurodevelopmental outcome $(2,3)$. Accompanying the quantitative surge of cortical brain growth is a dramatic change in the appearance of the fetal brain from the smooth contours seen at 24 wk of gestational age to the multiple convolutions that typify the mature brain. The timing of appearance and developmental changes of the cortical sulci and gyri have been outlined from anatomic studies (4-6). One of the cortical sulci detailed on neuroanatomic specimens is the cingulate sulcus which develops in the periventricular region of the cerebral hemispheres. This region is imaged routinely on neonatal cranial ultrasound studies to detect periventricular and intraventricular pathology. Because of its central location and developmental symmetry, the sequential imaging of the cingulate sulcus allows for a reproducible noninvasive assessment of cerebral sulcal

Received May 16, 1989; accepted July 28, 1989.

Correspondence Terri A. Slagle, M.D. Assistant Clinical Professor of Pediatrics, Children's Hospital of San Francisco, Department of Neonatology, 3700 California St., San Francisco, CA 94118.

Supported by the Children's Miracle Network Telethon.

' Presented in part before the Society for Pediatric Research, Washington, DC, May 1987 configuration and maturation and thus a qualitative assessment of brain maturation.

The purpose of our longitudinal study was to define the normal pattern of cingulate sulcus development in utero and to compare fetal development with postnatal development in infants born preterm. Additionally, we wished to determine whether brain injury, such as intracranial hemorrhage or periventricular leukomalacia, was associated with alterations in the maturation of the cingulate sulcus.

\section{MATERIALS AND METHODS}

Patients. Two groups of study infants were selected prospectively from among all infants admitted to Crouse Irving Memorial Hospital from July 1,1985 to June 30,1986 . One group was drawn from all 165 infants who were between 24 and 32 wk of gestational age. Thirteen infants died before $72 \mathrm{~h}$ of age and two parents refused informed consent; the remaining 150 infants were enrolled in the study. Gestational age for these infants was determined from maternal dates of the last menstrual period. Gestational age was substantiated by early second trimester ultrasound examinations in 127 cases $(85 \%)$ and by early prenatal visits in an additional 21 cases $(15 \%)$. Gestational age of all infants beyond 28 wk was confirmed by Dubowitz examination (7). There were only two instances where maternal dates were unavailable and Dubowitz examination alone was used to determine gestational age. A second group consisted of 61 infants who were selected at random from the infants of 33 to $40 \mathrm{wk}$ of gestational age. Gestational age assessment was determined in the same manner as in group one infants. None of the infants in study group two had clinical or ultrasonographic evidence of CNS abnormality.

Ultrasonography. Serial cranial ultrasounds were performed on all infants in group one at $48 \pm 24 \mathrm{~h}$ of age, at 7 and $14 \mathrm{~d}$ of age and then biweekly until 40 wk of postconceptional age. Infants in group two had a single cranial ultrasound within $72 \mathrm{~h}$ of birth. Uitrasounds were obtained in the nursery using a real time ultrasound scanner (ATL Mark 100, Advanced Technology Laboratories, Bothell, WA) with both 5 and $7.5 \mathrm{mHz}$ sector transducers. Sequential images were taken through the anterior fontanelle in the parasagittal and coronal planes. Ultrasounds were interpreted by two examiners for evidence of hemorrhage, ventricular size, periventricular echo pattern, and configuration of the cingulate sulcus. Intracranial hemorrhage was graded by the extent of hyperechoic regions and the result of hemorrhage on ventricular size. The location, density, symmetry, and persistence of periventricular echoes were evaluated on all scans. Asymmetric periventricular echo patterns seen on both parasagittal and coronal views or a pattern of echogenicity equal to that of the choroid plexus were classified as periventricular leukomalacia (8). Brain insult was considered mild if hemorrhage was isolated to the germinal matrix or if a small amount of blood 
was present in the ventricles. Insult was defined as severe if hemorrhage distended the ventricular system or extended into the brain parenchyma, or if periventricular leukomalacia was noted.

The cingulate sulcus was identified on the parasagittal views in the region between the thalamus and the anterior fontanelle. Evaluation of cingulate sulcus configuration was made in the region anterior to the atrium of the lateral ventricle. When unilateral hemorthage or periventricular leukomalacia was noted, the contralateral hemisphere was used to stage cingulate sulcus development. In addition, the combined depth of the left and right cingulate sulci on coronal images taken at the level of the foramen of Monro was measured using calipers.

Prenatal cingulate sulcus development was based on the appearance of the sulcus on the initial cranial ultrasound obtained in all 211 study infants. The postnatal pattern of cingulate sulcus development was established from the serial cranial ultrasounds obtained from birth to $40 \mathrm{wk}$ postconceptional age in the infants of $\leq 32$ wk gestational age (group one). Finally, a comparison of postnatal cingulate sulcus development was made between the group one infants with normal cranial ultrasounds and those with evidence of severe brain insults.

Statistical analysis. The difference between prenatal and postnatal cingulate sulcus development was delineated by $x^{2}$ analysis with Yates correction. Comparisons between cingulate sulcus maturation and assessments of brain insult were made with a one way analysis of variance followed by a Student's NewmanKeuls test for multiple comparisons. All data are presented as mean $\pm 1 \mathrm{SD}$. This study was approved by the hospital research review committee and parental informed consent was obtained before patient enrollment.

\section{RESULTS}

The gestational age distribution of the 150 study group one infants is shown in Figure 1 . Seventy-one infants $(47 \%)$ were $\leq 28$ w of gestational age, $86(57 \%)$ were male, $125(83 \%)$ were inborn and $127(85 \%)$ were white. A total of 30 infants $(20 \%)$ had ultrasonographic evidence of brain insult. These included nine infants with mild insults (six with isolated germinal matrix hemorrhage and three with small amounts of ventricular blood) and 21 infants with severe brain insults (11 with ventricular bleeding associated with ventriculomegaly, four with parenchymal extension of intraventricular hemorrhage, and six with periventricular leukomalacia). A total of 144 infants $(96 \%)$, including 27 with brain insults, survived to $40 \mathrm{wk}$ of postconceptional age.

The cingulate sulcus was first identified on cranial ultrasound

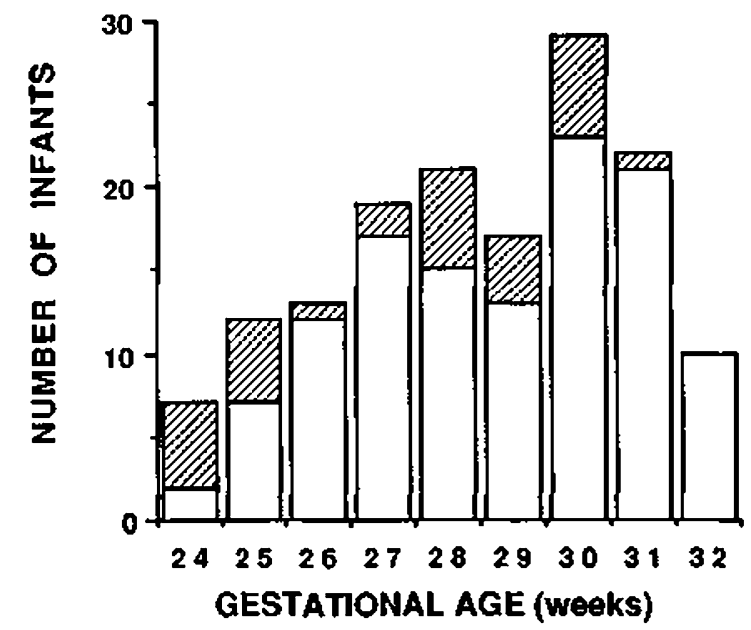

Fig. 1. Gestational age distribution for 150 study infants. Hatched areas represent infants with intracranial hemorrhage or periventricular leukomalacia. examinations as one or more discontinuous echoes at a mean of $26 \mathrm{wk}$ of gestational age. During the subsequent $14 \mathrm{wk}$, the cingulate sulcus followed a predictable sequence of changes (Fig. 2). At a mean gestational age of $30 \mathrm{wk}$, the sulcus appeared as a single continuous linear echo (continuity). Over the next 4 wk, perpendicular echoes off of the primary sulcus (branching) were identified. Finally, a pattern of branches from the cingulate sulcus merging with other cortical sulci (cobblestone) was seen at term. An increase in the depth of the cingulate sulcus was found in association with more complex branching patterns. The combined length of the left and right cingulate sulcus increased progressively, averaging $4 \pm 3,9 \pm 3,12 \pm 2$, and $15 \pm 3 \mathrm{~mm}$ at $28,32,36$, and $40 \mathrm{wk}$ of postconceptional age, respectively.

The timing of appearance of each stage in cingulate sulcus maturation is depicted in Figure 3. The pattern and postconceptional timing of prenatal maturation (determined from the initial ultrasound on each infant) was similar to postnatal maturation (determined from the sequential ultrasounds from birth to 40 wk postconceptional age). Furthermore, cingulate sulcus maturation was not related to gestational age at birth. Infants born at 24 to $26 \mathrm{wk}$ of gestational age and those born at 30 to $32 \mathrm{wk}$ of gestational age, for example, had similar timing of cingulate continuity $(30.9 \pm 3.0$ vs $31.5 \pm 1.4$ wk postconceptional age, respectively), first sulcus branch $(31.9 \pm 2.6 \mathrm{vs} 32.4 \pm 1.8 \mathrm{wk})$ and multiple sulcus branches $(34.2 \pm 2.7 \mathrm{vs} 35.0 \pm 2.0 \mathrm{wk})$.

Infants with ultrasound evidence of brain insult showed significant delays in postnatal development of the cingulate sulcus. These delays in qualitative brain growth were attributable solely to those observed in the infants with intraventricular hemorrhage complicated by ventriculomegaly or parenchymal extension and periventricular leukomalacia (Fig. 4). These infants with severe brain insults demonstrated significant delays compared to infants without brain insult for cingulate sulcus presence $(29.5 \pm 1.9$ vs $28.5 \pm 1.4 \mathrm{wk}$ postconceptional age, respectively, $p<0.05$ ) sulcus continuity $(32.9 \pm 3.1$ vs $31.3 \pm 2.0 \mathrm{wk}, p<0.01)$, appearance of first sulcus branch $(34.0 \pm 2.5 \mathrm{vs} 32.2 \pm 1.8 \mathrm{wk}$, $p<0.01)$, and multiple branches $(36.1 \pm 2.4$ vs $34.7 \pm 2.1 \mathrm{wk}$, $p<0.01$ ). A critical evaluation was made to determine whether mechanical or technical factors contributed to the observed delays in cingulate sulcus maturation seen in infants with severe brain insult. Fifteen of the 18 infants had unilateral brain insults and demonstrated no asymmetry of sulcus development between the injured and contralateral hemisphere. The timing of appearance of cingulate sulcus landmarks for infants with mild brain insult (isolated germinal matrix hemorrhage or intraventricular hemorrhage without ventriculomegaly) was similar to that for infants with normal cranial ultrasounds.

\section{DISCUSSION}

Neuroanatomic studies have demonstrated dramatic changes in the developing cerebral hemispheres during the last intrauterine trimester (4-6). The cingulate sulcus is anatomically evident at $20 \mathrm{wk}$ of postconceptional age as a discontinuous, shallow indentation on the medial aspect of the brain. As with all cortical sulci, the cingulate gradually increases in depth over several weeks, becoming a well demarcated fissure by 26 wk of gestational age. Thereafter, primary, secondary, and tertiary branches of the primary sulcus increase in number and depth. In our study, we used ultrasonography to prospectively detail these qualitative changes. The sequence of events depicted by sonograms correspond well to the development established from anatomic studies (4-6).

The identification of cortical gyri and sulci on ultrasonographic images has been reported previously $(9,10)$. Worthen et al. $(10)$ were able to retrospectively correlate gestational age with three sonographic stages of sulcal development. The earliest stage, in which primary sulci appeared as straight lines, was typical of the most immature infants (gestational age, $26 \pm 2 \mathrm{wk}$, mean \pm SD). 